\title{
«Una gestazione lunga e travagliata»: la fondazione delle Università di Sassari e Cagliari
}

\section{«A long Way and troubled Gestation»: The Foundation of the Universities of Sassari and Cagliari}

ELEONORA TODDE

eleonora.todde@unica.it

Università degli Studi di Cagliari

Riassunto: Il lento iter di fondazione delle Università di Sassari e di Cagliari vide impegnate le due città sarde per l'ottenimento di una scuola di istruzione superiore per i propri giovani. La lunga gestazione fu il frutto di numerose richieste parlamentari e spasmodiche ricerche di finanziamenti che portarono, attraverso una lotta continua per la supremazia verso il riconoscimento di prima università del regno e i controversi rapporti con la Compagnia di Gesù, al primo passo verso una istruzione di tipo accademico. Il presente contributo ricostruisce il processo di formazione delle università dalle prime istanze poste in seno al Parlamento presieduto dal viceré Antonio de Cardona fino ai privilegi reali della prima metà del XVII secolo.

Parole chiave: fondazione, università, Sardegna, Cagliari, Sassari

\begin{abstract}
The slow process of foundation of the University of Sassari and Cagliari saw the two Sardinian cities committed to obtaining a higher education school for their young people. The long «gestation» was accompanied by numerous parliamentary requests, the spasmodic search for funding, the competition for recognition as the first university in the kingdom and the relationship with the Society of Jesus, which represented the first step towards an academic education. This contribution reconstructs the process of university education from the first demands made in the Parliament chaired by Viceroy Antonio de Cardona to the royal privileges of the first half of the 17 th century.
\end{abstract}

Keywords: foundation, university, Sardinia, Cagliari, Sassari 


\section{Introduzione}

L'istruzione sarda vide un progressivo incremento nelle principali città dell'isola a partire dalla seconda metà del XVI secolo, in seguito alla fondazione dei collegi gesuiti e al costante interessamento delle amministrazioni comunali circa il funzionamento del sistema scolastico. Gli sforzi profusi non si limitarono solamente alla ricerca di fondi per il mantenimento dei collegi, ma si indirizzarono verso un ampliamento delle discipline impartite e un miglioramento della qualità dell'insegnamento (Turtas 1985-1986). Ben presto l'esigenza di una istruzione di livello accademico si fece sentire e iniziarono le prime mobilitazioni per la nascita di una università in Sardegna. L'iter di fondazione di entrambi gli atenei sardi, visto come una «gestazione lunga e travagliata» (Nonnoi 2016: 7), è durato circa 70 anni, ovvero dalla prima richiesta durante il Parlamento del viceré Antonio de Cardona del 1543 fino al privilegio di fondazione dell’Università di Cagliari del 31 ottobre 1620.

Fin dalle origini, però, le università di Cagliari e Sassari gareggiarono fra di loro per ottenere il riconoscimento del primato di generalis Universitas Sardiniae, ricercando tra i documenti sulle origini dei due atenei la legittimazione di questo titolo. Purtroppo, la scarna documentazione sull'argomento e interpretazioni talvolta campanilistiche hanno portato a fraintendimenti e retrodatazioni sulla vera nascita dell'istruzione universitaria nell'isola: Sassari ha infatti festeggiato i 450 anni dalla fondazione nel 2012, mentre Cagliari si accinge quest'anno a festeggiare i 400 anni. Le celebrazioni sono basate realmente sulla nascita degli atenei oppure si riferiscono a enti giuridicamente differenti? Per rispondere a questo quesito bisognerà ripercorrere le vicissitudini dei due atenei a partire dalla metà del Xvi secolo.

\section{La prima richiesta parlamentare}

Nel 1543, a Cagliari, si celebrò il Parlamento presieduto dal viceré Antonio Folch de Cardona durante il quale giunse per la prima volta la richiesta per l'apertura di una università in Sardegna. La prima istanza giunse il 16 settembre dal rappresentante della città di Cagliari che, con una richiesta meno articolata e municipalistica rispetto a quella di Sassari, come si vedrà più avanti, si preoccupò di porre l'attenzione sulla necessità di un generale innalzamento culturale dell'intero regno.

\footnotetext{
Ítem, com molts fadrins y jovent, los quals, havent-hi studi, entindrien en studiar y esser persones de lletres, se dexen del studi y de entendre en les lletres per falta de mestres y persones doctes, y, havent-hi studi, no faltaran mestres y doctors per llegir, que per ço, et alias, considerada la hutilitat se'n resultarà y honor de la ciutat y de tot lo regne, aprés del servey de nostre senyor Deu, y de sa magestat, sia statuït y ordenat se face estudi general en Càller ab certa renda, perquè no falten los mestres o doctors per a llegir ordinàriament, y que s prenga alguna quantitat del Parlament per ha que se faça, es mantenga dit estudi y la ciutat ajudarà de alguna cosa, lo que bonament porrà sols que tant bé no rest sense fer-se (ASCa, AAR, Parlamenti, vol. 158, c. 165v; Lattes 1909: 138).
} 
La richiesta di elevare Cagliari a sede universitaria venne inserita all'interno di un più ampio piano riformatore che prevedeva anche la richiesta di rendere obbligatoria per tutte le otto diocesi dell'isola l'istituzione di una scuola di grammatica, garantita dalle rendite delle mense episcopali e capitolari (ASCa, AAR, Parlamenti, vol. 158, c. 172r). La presenza di una scuola di grammatica in tutte le sedi di diocesi fu prescritta in precedenza durante il Concilio Lateranense IV del 1215 e ribadita, per la Sardegna, nel sinodo di S. Giusta del 1226, dove si propose l'istituzione alle sole sedi metropolitane, chiaro segno dell'arretratezza della cultura nell'isola (Conciliorum 1973: 240; Di Martino 1997).

Il 19 settembre, a pochi giorni di distanza dalla domanda di Cagliari, il rappresentante della città di Sassari chiese l'apertura di una sede universitaria nella città turritana e propose che il finanziamento, stimato in circa 400 ducati, fosse reperito principalmente dalla somma a disposizione del Parlamento e la restante parte dall'arcivescovo, dalla stessa città e da alcuni privati cittadini.

\begin{abstract}
Més, vist y ab experiència conegut, que los locs y ciutats són tant més nobilitadas y decoradas quant los abitadors y ciutadans de aquellas són de major experiència, letras, doctrina en diversas facultats y sièncias, y com las despesas, costas grandíssimas y perhils grans de anar en los estudis generals destorbe a molts de no seguir aquells en apendre letres, teologia, filosofia y medisina y altres facultats, no obstant que aquels tingan bons principis, segons tenen alguns en la dita ciutat de Sàser, los quals per defecte de facultats o compliment de aquellas no poden acudir, se supplica esser concesa alguna cantitat per a fundar en dita ciutat un estudi general, encara que los propris ciutadans o universitat y donàs alguna cosa o altrament, y así matex que lo senyor arquebisbe y donàs una part de beneficis sine cura in tantum que, computatis omnibus, se obtingués una suma de quatre cents ducats y més y manco, segons fos miglior vist per lo servey de nostre senyor Deu, y de la real magestat, bé y readrés essencial de dita ciutat y ciutadans de aquella (ASCa, AAR, Parlamenti, vol. 158, cc. 182r-183v; Lattes 1909: 139).
\end{abstract}

Il rappresentante espresse la convinzione che l'università fosse lo strumento più idoneo per garantire il miglioramento e il ricambio della classe dirigente cittadina. A sostegno di tale richiesta vennero posti all'attenzione del viceré alcuni ostacoli che gli studenti sardi, desiderosi di conseguire una istruzione universitaria, dovevano affrontare in quel periodo: la lontananza da casa, le ingenti spese per le famiglie, i pericoli del mare -allora popolato da personaggi ostili- e la mancanza di comunicazione tra la Sardegna e il mondo esterno (Sorgia 1963: 92).

Entrambe le città volevano garantire ai propri intellettuali locali una possibilità di accesso alle cariche e agli uffici che esigevano una formazione di livello superiore, spesso affidati a notabili stranieri, avvantaggiati per i titoli di studio conseguiti. Ad ogni modo, se è pur vero che la città del sud dell'isola godesse di condizioni più favorevoli e maggiori probabilità di successo rispetto a Sassari, la popolazione scolastica di entrambe le città non superava il centinaio di unità e la qualità dell'insegnamento fino ad allora impartito risultava mediocre. 
Probabilmente le richieste poste in seno parlamentare esulavano dalle competenze o dagli abituali campi di interesse del viceré il quale, in prima battuta, si limitò a evidenziare come la domanda andasse riformulata dai tre stamenti reale, militare ed ecclesiastico per poi essere successivamente presentata in forma congiunta al sovrano. La petizione di Cagliari fu esaminata dal futuro re Filippo II, allora reggente dei regni iberici al posto di Carlo V, solamente nel 1545. In quella occasione il rappresentante cittadino mise in evidenza come l'istituzione sarebbe stata di «grande servizio per Nostro Signore e per sua maestà e di universale beneficio per tutto quel regno». Filippo ordinò al viceré di esaminare la questione con gli stamenti e di riferire a corte quanto discusso, in modo che il sovrano fosse nelle condizioni di prendere una decisione. Purtroppo, non si hanno fonti in merito alla petizione sassarese, sicuramente non dissimile da quella dell'altra città sarda, e alla relazione del viceré: la questione dell'università si trascinò fino al parlamento successivo, presieduto dal viceré Lorenzo Fernàndez de Heredia.

Contemporaneamente, un impulso che indirettamente contribuì a favorire la fondazione di uno studium fu rappresentato da un provvedimento emanato da Filippo II nel quale si vietò ai giovani sardi l'iscrizione in università non iberiche, allo scopo di evitare un possibile contatto con le dottrine della riforma protestante. Nonostante il quadro politico e religioso dell'Europa nel periodo della controriforma avesse creato questa situazione di chiusura anche per la Sardegna, tale provvedimento regio si rivelò in realtà vantaggioso per la costituzione dell'università nell’isola.

\section{Il parlamento del viceré Lorenzo Fernandez de Heredia}

La grave situazione degli studi e la preoccupazione di una crisi religiosa avevano portato il principe reggente Filippo a premere verso la costituzione di studi generali in modo che si formassero «maestros de buena y sana doctrina» (Putzolu 1955). Il reggente inviò una lettera all'allora viceré Lorenzo Fernandez de Heredia nel quale ordinò che il parlamento votasse una apposita dotazione finanziaria per l'erezione di uno studio generale, assicurando un aiuto imperiale qualora ci fosse una congrua disponibilità al successo dell'iniziativa. Questa missiva rappresentò il primo intervento del governo spagnolo sull'istruzione universitaria. Il viceré si rivolse anche agli amministratori di Cagliari e agli stamenti, oltre al generale dei francescani affinché inviasse alcuni frati nel convento cagliaritano con lo scopo di aprire un collegio: le lezioni non si sarebbero rivolte solo ai religiosi ma anche a uditori laici, istituendo di fatto uno studium di istruzione più elevato. Il viceré, per dimostrare il suo intento, stanziò la cifra di 120 ducati annui al convento francescano di Cagliari per il mantenimento degli insegnanti (AGS, Estado, Legajos 312, c. 514r).

Nel frattempo, i tre stamenti, in ottemperanza a quanto prescritto loro durante il precedente parlamento, presentarono una petizione congiunta mediante la quale si mostrarono particolarmente preoccupati della situazione svantaggiosa che gravava su alcuni meritevoli giovani che, per motivi economici, non potevano proseguire gli studi. La rinnovata petizione aveva, infatti, tra le sue più 
importanti motivazioni il fatto che sull'isola vi fossero molti giovani desiderosi di poter completare gli studi, impossibilitati a partire.

Sassari non aveva un proprio rappresentante in quel parlamento, pertanto la richiesta degli stamenti ebbe per oggetto la fondazione di una università con sede a Cagliari, dove il vescovo Baldassare de Heredia metteva a disposizione i locali presso la chiesa di Santa Croce, in aggiunta a una somma di denaro per il suo mantenimento.

\begin{abstract}
Ítem, supplícan dits staments com de totes le virtuts excel lesca la doctrina y per aquella les terres y regnes són ben regits y governats, de la qual esta ciutat de Càller y regne de Serdenya stà molt probe y fretureja, y com hi haja molts jóvens y fadrins que àman les letres y se darían ad aquelles si hi hagués comoditat de studiar, lo que no poden a causa que han de anar fora del regne y han mester quiscun any qui ls provehesca de dinés per sustentació llur, y per ser molt probes com són no poden proseguir ni anar; de hont se segueix recaure tanta ignoràntia com cau en les persones ecclesiàstiques y curats de fora y altres persones layques, lo que no seria si hi hagués algun studi general; e com lo reverendíssimo arquebisbe de Càller, don Balthazar de Heredia, prevehent lo dany e ignoràntia que per falta de dit studi general recau en los regnícoles y compatriotas de aquest regne s'és offert en fer y fundar hun studi general en Sancta Creu y per dit effecte se li ha dat una casa, y per que ab més fervent ànimo lo dit arquebisbe se esforçe en fer y fundar dit studi, supplica per ço a vostra il lustríssima senyoria, lo qual presideix en nom de sa magestat en lo present Parlament, que de les pecúnies del present Parlament sia presa una suma condecent de la qual dit studi se puga dottar, la qual servesca per renda per sustentació de les persones que en dit studi residiran per lo tant de bé y útil que de semblant cosa tant sacra y bona ne resultarà al servey de Deu omnipotent y de sa magestat y ben de aquest seu regne y regnícoles de aquell (ASCa, AAR, Parlamenti, vol. 160, cc. 205v, 219v-220v; Sorgia 1963: 83-84).
\end{abstract}

Durante le sessioni del nuovo parlamento, Ludovico de Cotes, vescovo di Ampurias e Civita, presentò, mediante il suo procuratore Antonio Corbo, una petizione nella quale sottolineò la pressante esigenza di una università in Sardegna, pur non indicando una preferenza per la sede (ASCa, AAR, Parlamenti, vol. 160, cc. 394r, 397r-v; Sorgia 1963: 203-204). L'approvazione degli atti durò a lungo e siamo a conoscenza della risposta della principessa Giovanna d'Austria, reggente per il fratello Filippo, allo stamento ecclesiastico, dove si sollecitò il mantenimento della promessa fatta dall'arcivescovo di Cagliari per proseguire l'iniziativa. Come si è detto in precedenza, a fronte della scarsità demografica dell'isola, fu previsto un unico studium per tutta la Sardegna, difatti un'ulteriore causa di rallentamenti nella sua nascita fu costituita dal contenzioso sorto tra le città di Cagliari e Sassari, entrambe desiderose di averne uno.

Sia Cagliari che Sassari cercarono, quindi, altre soluzioni, in particolare presso la Compagnia di Gesù. Già nel 1552 l'arcivescovo turritano Salvatore Alepus richiese la presenza di quattro gesuiti che si dedicassero alla predicazione, all'istruzione del clero e all'insegnamento della grammatica e della dottrina cristiana ai fanciulli; in cambio prometteva una rendita di 100 scudi annui. Queste trattative 
non ebbero, però, inizialmente successo (Turtas 1988: 26-27). Si dovette aspettare dieci anni per l'apertura delle prime classi nel collegio gesuitico di Sassari, mentre a Cagliari questo si inaugurò nel 1565. La presenza di queste scuole non portò all'accelerazione dell'iter di fondazione dell'università, bensì ne dilatò $i$ tempi poiché introdusse nel panorama formativo delle due città una valida alternativa e assorbì una parte degli interventi finanziari regi a favore dell'istruzione (Turtas 1986).

\section{L'approvazione pontificia e i privilegi reali di fondazione}

L'effettiva fondazione di uno studium generale per la Sardegna avvenne infine, in seguito a una terza richiesta, presentata dagli stamenti durante il Parlamento presieduto dal viceré Antonio Coloma, conte di Elda, il 3 marzo 1603. Nel 1573 Cagliari, infatti, richiese la trasformazione del collegio gesuitico in vera università: tale richiesta non venne però accolta. La nuova istanza, al contrario, ignorò completamente l'esistenza dei gesuiti e il loro apporto didattico e culturale, concentrandosi sulla istituzione di una università nella quale esercitare il pieno controllo. La petizione venne presentata in maniera congiunta dai tre stamenti e fu corredata da un preciso piano di finanziamento autonomo nel quale lo stamento ecclesiastico partecipava alla metà della copertura economica mentre l'altra metà era assicurata dalla stessa città.

\footnotetext{
Considerant los tres estaments de aquest regne de Sardenya, per orde de sa magestat congregats en estes reals generals Corts per a tractar y procurar tot lo que és servey de nostre Senyor y de sa real magestat e lo bé, augment y llustre del dit regne, de quanta importànsia sien per a conseguir tots los prementionats fins de les lletres y lo exersisi de elles, y que mediant les sciènties se conserva, confirma y exalta la santa fe y chatòlica doctrina de la qual, més que de altra cosa, se pot molt honorar y honrar aquest dit regne per haver-se en el conservat sens infectió de error ni heregia des del temps dels apòstols, havent-la en el predicada hu dels 12 discebles de Christo, senyor nostre, y considerant que ab elles se cultivan los ingenis y que totes les cosses que en una repúbblica y regne ben governat son menester se alcànsan y se pòssan en son lloch y tenen lo llustre de que les històries estan plenes, y que per medi de lles matexes lletres y sciènties és estat y és tant il lustrat lo món, y que no hi ha agut ni hay província ben governada y de policia que no les àgia procurades y abraçades, e considerant axí bé y veent per esperièntia que aquest dit nostre regne, per dexar los demés, és estat y és notòriament il lustrat y millorat des de que com fins ara millor se ha pogut han emprés en ell ab nou fervor los estudis y exercissi de aquelles, ha paregut que se li restava alguna esperansa per aver-lo de tornar a 1'antich llustre que en temps dels romans particularment tenia, se podria sols alcansar ab instituir en dit regne un Estudi universal y publich com en tots los demés regnes se veu ser instituhit y fundat, en lo qual se ellegissen totes les facultats, tenint per molt sert que ab notable vantàgia pendria per ha si lo dit regne milloria, y que com se ha vist en ell lo profit ab cultivar-se més la terra se veuria millor ab la coltura dels enteniments dels quals és tant copiós que no se entén ser en res inferiors los subjectes que d'ell se venen en les Universitats de Espanya e Itàlia als dels altres regnes y províncies, de la dita santa y utillosa obra se'n servirà sumament nostre Senyor Deu per que se desterrarà per ella la ignorància centina de pecats y de mals y se guariran moltes ànimes que ab ella de cada dia se perden, se'n servirà també molt sa magestat per que tindrà aquest son regne fidelíssim major llustre y nova
} 


\begin{abstract}
forma y serà més enriquit y embellit de moltes cosses que en ell se poden fer quals se dèxan de posar per obra per no haver-i qui les promoga y porte a llum com convé per falta de art y de sièntia; tindrà també sa magestat més subjectes y persones de lletres y de vallor de les quals en qualsevol ocasió y temps se podrà millor y més servir lo patrimoni etiam de sa magestat, y axí bé tot lo regne anirà ab açò en tant gran augment que 's podrà dir haver-se-li dat nou ser y nova vida, ultra que se aforraran los particulars d'ell lo que gàstan, havent de trametre sos fills a lles Universitats fora del regne, no sens perdi axí de la vida com dells costums, màxime havent-los de enviar en Itàlia com los de més ho fan, puix les poques facultats que tenen no abastan per aver-los de provehir y enviar-los en Espanya com la magestat del rey nostre senyor, que està en lo sell, lo volia y pretenia, prohibint expresament com prohibí que no anassen a estudiar als de Itàlia. Per lo que et alias supplícan a vostra senyoria los dits estaments ecclexiàstich, militar y real que se servescha vostra senyoria il lustríssima en persona de sa magestat decretar que se puga y dega instituhir y fundar un Estudi y Universitat pública per als regnícols de esta isla y altres que a ella acudiran, y que 's fassa y mantinga en esta ciutat de Càller, en la qual àgian de legir totes les facultats, comensant des de ara per les superiors y més necessàries, y en la qual se púgan graduar com en les altres Universitats se acostuma tots los qui vuy saran y estudiaran en elles ab les gràcies, mercès, privilegis e immunitats que les altres Universitats de sa magestat de la Corona de Aragó tenen. Que de no, ultra lo servici de nostre Senyor y de sa magestat, ne restaran tots los dits estaments y en general tot lo regne ab perpetua obligatió a vostra senyoria il lustríssima y a sa casa que omni meliori modo et licet etc. (ACA, Consejo de Aragón, registros, Camára, 377, cc. 492-493; Doneddu 2015: 719-721)
\end{abstract}

Sassari si trovò impreparata e avanzò la medesima proposta, senza aver studiato un apposito piano finanziario e chiedendo che fosse il regno ad accollarsi la spesa per l'erezione dello studium. Tra le due richieste, solo quella cagliaritana ottenne l'attenzione regia e fu difatti accolta favorevolmente dal sovrano Filippo III il quale si riservò la sovraintendenza ed il patronato del nuovo istituto.

L'erigenda università dovette fin da subito fare i conti con due ordini di difficoltà: un primo problema era di natura finanziaria, dal momento che, fra i tre Stamenti che avrebbero dovuto farsi carico delle spese di manutenzione e gestione dell'ateneo, soltanto la prima voce dello stamento reale, ovverosia la città di Cagliari, offrì effettivamente il proprio supporto; una seconda criticità, invece, era rappresentata dalla carenza di personale docente qualificato, per ovviare alla quale vennero intrapresi dei negoziati tra la città di Cagliari e la compagnia di Gesù (Putzolu 1952-1953: 5-8).

Soltanto il 12 febbraio 1606 giunse l'approvazione pontificia, attraverso l'emanazione di un'apposita bolla da parte del papa Paolo V Borghese; secondo quanto previsto dal documento, l'arcivescovo cagliaritano assommò su di sé entrambe le cariche di rettore e di cancelliere, ottenendo in questo modo la piena giurisdizione sull'intero personale accademico. Ad un apposito Collegio, composto dall'arcivescovo, da un canonico del Capitolo della Cattedrale, da un delegato del Braccio militare e dal Consigliere fu affidato, invece, il potere esecutivo, ovverosia il compito di sorvegliare sulla effettiva applicazione degli statuti e sulle nomine dei docenti (ASCCa, Sezione antica, vol. 21; Pinna 1931; D’Arienzo 1997). Il documento prefigurava un progetto di uno Studio generale all'interno del quale sarebbero stati offerti gli insegnamenti di Lettere latine, greche, ebraiche, Arti liberali, 
Medicina, Filosofia e Scienze, secondo il modello delle Università di Salamanca, Valladolid e, in particolar modo, Lleida (Ferrante 2013).

La situazione rimase immutata fino al 1611, quando il visitatore generale del regno Martin Carrillo, alla fine della sua ispezione agli affari del regno, osservò la grave ignoranza che attanagliava la società isolana, constatando come i sardi avessero un bisogno «de doctrina y sciencias» e che gli studenti fossero costretti a recarsi fuori dall'isola per ricevere un'istruzione superiore, col grave rischio di essere contaminati dalle dottrine ereticali. Nella propria relazione, inoltre, Carillo suggerì che, istituendo una università sull'isola, si sarebbe ovviato al cronico problema dell'assenza di personale qualificato che, in precedenza, era necessario importare con maggiore dispendio economico da altre parti del regno.

Intanto Sassari non si era persa d'animo e, grazie ai dispositivi testamentari di Gaspare Vico e di Antonio Canopolo a favore del collegio gesuitico, le aule vennero ampliate in vista di nuovi insegnamenti. Nel 1612 il generale Claudio Acquaviva inviò al rettore del collegio sassarese il diploma nel quale si autorizzò il conferimento di gradi accademici in Teologia e in Filosofia agli studenti non gesuiti: in tal modo il collegio divenne, di fatto, una «università» di diritto pontificio. Il 9 febbraio 1617, il collegio gesuitico ottenne il diploma da Filippo III nel quale si accordò il riconoscimento regio ai gradi di Filosofia e Teologia, facoltà fino ad allora attivate (Turtas 1988: 158-162).

I ritardi nello stanziamento dei fondi e la crescita del collegio gesuitico di Sassari portarono, nel dicembre del 1619, i procuratori dei tre stamenti verso una nuova istanza al re, nella quale lo si supplicava di rendere operativo il disposto del 1603 per l'ateneo di Cagliari. Dopo un anno, il 31 ottobre 1620, arrivò il privilegio reale di fondazione, sottoscritto dal sovrano Filippo III. A differenza di quanto previsto dalla bolla pontificia del 1607, all'arcivescovo di Cagliari venne riservato il solo ruolo di cancelliere, con la facoltà di nominare un pro-cancelliere in sua assenza. Il rettore, invece, nominato ogni tre anni dai Consiglieri della città, fu scelto fra gli ecclesiastici laureatisi in Teologia o in Utroque iure: chi ricoprì questa carica, secondo il modello delle altre università catalano-aragonesi, divenne il più importante punto di riferimento per la vita accademica (D’Arienzo 1997).

Nonostante ciò, fu soltanto alla fine del 1623 che l'ambasciatore cagliaritano presso la corte di Madrid riuscì ad ottenere dal sovrano l'obbligo di compartecipazione finanziaria da parte di tutte le componenti del regno. Durante il parlamento del 1624, presieduto dal viceré Giovanni Vivas, Filippo IV ordinò a quest'ultimo di imporre a tutti e tre gli stamenti di offrire il proprio contributo economico per la realizzazione dello studium generale a Cagliari. Tale richiesta, tuttavia, incontrò la ferma opposizione di Angelo Manca, rappresentante sassarese a Madrid, che provocò un ulteriore rallentamento nei lavori del parlamento (Turtas 1988: 163-165). Anche a causa di questa controversia, dal momento che era stato previsto un unico ateneo per tutta l'isola, Sassari non contribuì mai al finanziamento dell’Università di Cagliari. Secondo quanto previsto dagli accordi, gli 
stamenti militare ed ecclesiastico, come quello reale, avrebbero dovuto versare una somma di 2.000 ducati all'anno per la gestione, il funzionamento e le spese dell'università. Tuttavia, la questione finanziaria fu risolta, per quanto soltanto in parte, grazie all'intervento della città di Cagliari, che fin dall'inizio si fece carico degli oneri economici del nascente istituto, e alla donazione di 10.000 lire sarde del conte di Serramanna, Antonio Brondo, il 16 agosto 1624.

Le costituzioni dell'Università di Cagliari, che constano di 40 articoli, furono promulgate in data $1^{\circ}$ febbraio 1626 dal consigliere capo della città Giovanni Dexart e dai consiglieri civici Filippo Silvestre, Giovanni Giacomo Martis e Gerolamo Aleo. Il Dexart, dottore in Utroque iure, aveva dedicato i propri studi a diversi statuti universitari spagnoli e italiani, e scelse come modello per l'organizzazione dell'ateneo cagliaritano quella dell'Università di Lleida (Canepa 1925). L'università fu dotata di quattro facoltà: Teologia, Medicina, Leggi e Canoni e Filosofia e Arti; il corpo accademico era costituito da 54 membri, fra cattedratici, giunte di facoltà e collegi dei dottori.

Nel 1632 Geronimo de Homedes, consigliere capo e rappresentante di Sassari al Parlamento del viceré Bayona, supplicò il re per l'ottenimento di nuovi gradi accademici:

\footnotetext{
Ítem, supplica dit síndich a vostra senyoria il lustríssima se servesca suplicar a sa magestat de que, segons ha fet mèrit a la ciutat de Sàsser de poder-se graduar en theologia y filosophia, li fassa mèrit de dar llicència de que se púgan en ditta ciutat graduar en las demès facultats (ASCCa, Sezione Antica, Atti del Parlamento Pimentel, vol. 10, c. 855v; Tore 2008: 687).
}

Il re concesse al rettore, ai dottori, ai maestri e ai licenziati di Sassari il diritto di conferire gradi accademici anche in diritto civile, in diritto canonico e in medicina, «come si usa nelle università di Spagna e di Sardegna»: venne, pertanto, ampliato il privilegio concesso da Filippo III nel 1617 alle sole facoltà di Filosofia e di Teologia (Turtas 1988: 175).

\section{Conclusioni}

La competizione serrata tra $i$ due atenei sardi vide già dalle prime battute la volontà delle due città di ottenere il privilegio di fondazione per una nuova università. La richiesta di Sassari risultò sicuramente calibrata rispetto alla reale situazione cittadina, mentre quella di Cagliari presentò delle motivazioni di «ordine generale». Entrambe, però, commisero un errore nel presentare una istanza separatamente e senza tener conto della prassi parlamentare che pretendeva la richiesta congiunta dei tre stamenti. L'errore fu fatale perché procrastinò la richiesta fino al parlamento successivo.

Nel Parlamento del viceré de Heredia, Sassari apparve in netto svantaggio poiché non aveva nessun rappresentante durante i lavori: questo portò alla presentazione di una istanza a favore di Cagliari, appoggiata anche dall'arcivescovo cittadino che forniva mezzi e soldi a sostegno 
dell'erezione. Lungaggini burocratiche portarono le due città a cercare una soluzione che trovarono nella Compagnia di Gesù e nella nascita dei relativi collegi. Se in precedenza Cagliari sembrasse in vantaggio rispetto alla città turritana, così non si poteva affermare alla fine del XVI secolo quando il collegio sassarese funzionava a pieno regime.

Una nuova occasione di vantaggio di Cagliari si ebbe nel 1602, durante il Parlamento Coloma, nel quale il re approvò la nascita di una università nella cittadina; al contempo Sassari trasformò il proprio collegio gesuitico prima in una università pontificia nel 1611 e poi in una università statale nel 1617. Le suppliche di Cagliari si fecero esecutive nel 1620, quando il re Filippo III emanò il privilegio di fondazione della nuova università.

Trascorsero tuttavia sei anni prima che l'ateneo fosse operativo e la querelle tra le due città sarde si infiammò nel corso del 1627: l'Università di Cagliari, chiamata generalis Universitats Sardiniae, si considerava l'università del regno; l'arcivescovo di Sassari decise, quindi, di aggiungere il titolo di primariae Universitatis ac Studii generaliis, facendo infuriare l'arcivescovo e nuovo rettore dell'ateneo cagliaritano Ambrogio Machin (Battlori 1969). Solamente nel 1632 Sassari ottenne il privilegio reale per il conferimento dei gradi in tutte le facoltà. Appare chiaro come questa rivalità abbia fortemente contribuito al ritardo nella nascita di una istruzione universitaria in Sardegna e come abbia influenzato, negativamente, le complesse dinamiche tra l'isola e la corona.

Ancora oggi è difficile affermare quale sia la «prima» università sarda: nel 2012 l’Università di Sassari ha celebrato il $450^{\circ}$ anniversario prendendo, di fatto, come data di fondazione quella del collegio gesuitico (Università 2013), mentre Cagliari si accinge quest'anno a festeggiare i quattrocento anni dal privilegio di fondazione emanato da Filippo III. Si ritiene errato partire dall'istituzione del collegio gesuitico che, come affermato in precedenza, non rilasciava gradi accademici formalmente riconosciuti fino al 1617, data che invece segna, con assoluta certezza, l'istituzione giuridica dell'ente sassarese. In questo modo, il presente anno accademico segnerebbe il $403^{\circ}$ anno dalla nascita.

A ben ragione Cagliari prende come data fondante il 1620, anno del riconoscimento regio, benché l'emanazione delle costituzioni, l'inaugurazione del primo anno accademico e la vera «nascita» dell'ateneo siano da ricercarsi nel 1626. In tal modo Cagliari avrà una doppia numerazione: la prima riguardante la data di fondazione, la seconda gli anni accademici. 
Elena Todde. «Una gestazione lunga e travagliata»: la fondazione delle Università di Sassari e Cagliari

\section{Bibliografia}

Batllori, M. (1969), L’Università di Sassari e i collegi dei gesuiti in Sardegna: saggio di storia istituzionale ed economica, Milano, Giuffrè.

Canepa, M. (1925) «Le Constitutiones dell’Università di Cagliari», La Regione, II, n. 2, pp. 1-23.

Conciliorum oecumenicorum decreta (1973) a cura di Alberigo, G., Dossetti, G.A., Joannou, P.P., Leonardi, C., Prodi, P., Bologna, Istituto per le scienze religiose.

D'Arienzo, L. (1997) I documenti originali di fondazione dell'Università di Cagliari, Cagliari, Università degli studi di Cagliari - CIMAS.

Di Martino, E. (1997) Il codice di Santa Giusta della Biblioteca Universitaria di Cagliari, tesi di laurea, Facoltà di Lettere e Filosofia, Università degli Studi di Cagliari.

Doneddu, G. (2015) Il Parlamento del viceré Antonio Coloma conte di Elda (1602-1603), Acta Curiarum Regni Sardiniae, 13, Cagliari, Consiglio Regionale della Sardegna.

Ferrante, C. (2013) «Cagliari e Lerida, il modello di fondazione di uno Studio municipale: le Costituzioni del 1626», in Le origini dello Studio generale sassarese nel mondo universitario europeo dell'età moderna, a cura di Brizzi, G.P., Mattone, A., Bologna, CLUEB, pp. 61-73.

Lattes, A. (1909) «Per la storia delle Università sarde, Documenti nuovi», Archivio storico sardo, V, pp. 137-141.

Nonnoi, G. (2016) «Un ateneo in bilico tra sopravvivenza e sviluppo», in Rapetti, M. La riconquista dei saperi. Il pareggiamento dell'Università di Cagliari, Cagliari, Aipsa Edizioni, pp. 7-33.

Pinna, M. (1931) Gli atti di fondaz̧ione dell'Università degli Studi di Cagliari, estratto di Annuario della Regia Università degli Studi di Cagliari per l'anno scolastico 1931-32, Cagliari, Tip. P. Valdès.

Putzolu, E. (1952-1953) «La partecipazione dei Padri Gesuiti all'insegnamento nell’Università di Cagliari in un documento inedito del 1626», Studi sardi, XII-XIII, pp. 1-8.

—_. (1955) «Per la storia della Università sarda. Una sconosciuta iniziativa di Filippo II di Spagna», Nuovo Bollettino bibliografico sardo, I, fasc. 4, p. 9.

Sorgia, G. (1963) Il parlamento del viceré Fernandez de Heredia (1553-1554), Milano, Giuffrè.

Tore, G. (2008) Il Parlamento del viceré Gerolamo Pimentel marchese di Bayona e Gaspare Prieto, presidente del Regno, Acta Curiarum Regni Sardiniae, 17, Cagliari, Consiglio Regionale della Sardegna.

Turtas, R. (1985-1986) «Amministrazioni civiche e istruzione scolastica nella Sardegna del Cinquecento», Quaderni sardi di storia, 5, pp. 83-108.

1986) La Casa dell'Università. La politica edilizia della Compagnia di Gesù nei decenni di formażione dell'Ateneo sassarese (1562-1632), Sassari, Edizioni Gallizzi.

- (1988) La nascita dell'università in Sardegna. La politica culturale dei sovrani spagnoli nella formazione degli Atenei di Sassari e di Cagliari (1543-1632), Sassari, Dipartimento di Storia - Università degli Studi di Sassari.

SCRIPTA, Revista internacional de literatura i cultura medieval i moderna, núm. 15 / juny 2020 / pp. 229 - 240 
Elena Todde. «Una gestazione lunga e travagliata»: la fondazione delle Università di Sassari e Cagliari

Università degli studi di Sassari: 450 anni di storia. Anno accademico 2011-2012 (2013) a cura Comitato organizzatore per le celebrazioni del $450^{\circ}$ anniversario di fondazione dell'Università degli studi di Sassari, Muros, Nuova stampa color.

\section{Fonti archivistiche}

Archivio di Stato di Cagliari, Antico Archivio Regio, Parlamenti, vol. 158.

-, vol. 160.

Archivio Storico del Comune di Cagliari, Sezione antica, vol. 21.

- Atti del Parlamento Pimentel, vol. 10.

Archivo de la Corona de Aragón, Consejo de Aragón, Registros de la Real Camara de Aragón, n. 377.

Archivo General de Simancas, Estado, Legajos, n. 312.

SCRIPTA, Revista internacional de literatura i cultura medieval i moderna, núm. 15 / juny 2020 / pp. 229 - 240 